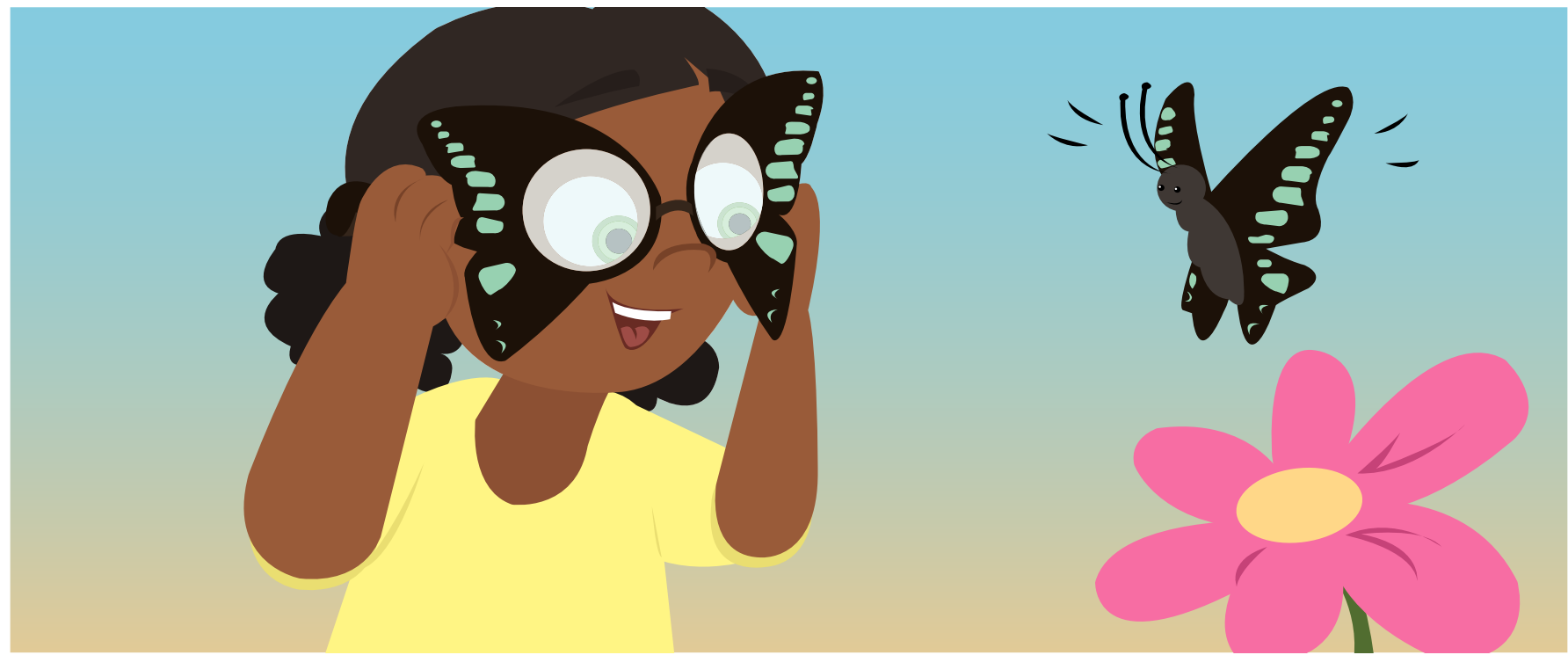

\title{
THE MORE, THE BETTER? A BUTTERFLY WITH 15 KINDS OF LIGHT SENSORS IN ITS EYE
}

\section{Pei-Ju Chen, Finlay J. Stewart and Kentaro Arikawa*}

Laboratory of Neuroethology, Department of Evolutionary Studies of Biosystems, SOKENDAl, Hayama, Japan

\section{REVIEWED BY:

MELVILLE
SENIOR HIGH
SCHOOL
AGE: $13-14$

\section{COLOR VISION}

The ability to detect differences in the wavelengths making up light, rather than just the brightness of the light.
Colors are everywhere in nature, and they communicate useful information. Flowers use colors to advertise that they have nectar, fruits change color when they are ripe, and birds and butterflies use their colorful wings to find mates or to startle enemies. To use this information, animals must be able to see colors. Humans have "trichromatic" color vision, meaning that all the colors we perceive can be produced by mixing three primary colors - red, green, and blue. This is because we have three kinds of light-sensing cells in our eyes, one kind sensitive to red, one to green, and one to blue light. Different species have different types of light-sensing cells. Honey bees also have three types, but they have cells that sense ultraviolet light instead of red light. Butterflies typically have 6 or more types of light-sensing cells, but we found one swallowtail species that has at least 15, which is the record among insects. In this article, we discuss how the world might look to a butterfly with such a complex eye.

\section{HOW DO WE KNOW IF ANIMALS CAN SEE COLORS?}

As humans, we are very dependent on color vision for our understanding of the world around us. Color is a property of light. Light travels in waves, 
LIGHT-SENSING CELL

(OR "PHOTORECEPTOR")

A special type of nerve cell found in animal eyes that produces electrical signals when light shines on it.

\section{FIGURE 1}

A. Color and wavelength of light. Light is made up of waves. For any wave, we can measure the wavelength, which is the distance between two neighboring wave peaks (that is, the length of one cycle). We see lights of different wavelengths as having different colors; shorter wavelengths are bluish while longer wavelengths are reddish. B. White light contains many different wavelengths. You can separate white light into its different wavelengths by passing it through a prism, or reflecting it off a special kind of surface. Compact disks (CDs) have this property, so you can make a rainbow with a flashlight and a CD. and the distance between two waves is called the wavelength. We perceive lights of different wavelengths as having different colors; long wavelengths look reddish and short wavelengths look bluish (Figure 1A). White light is made up of all wavelengths mixed together. You can see this when you look at a rainbow in the sky, or you can even demonstrate the effect yourself (Figure 1B): if you reflect light from a compact disk onto a blank surface, you will see the light split into different colors resembling a rainbow, or "spectrum." We call the range of wavelengths that we can see "visible light," and visible light has wavelengths from about $400 \mathrm{~nm}\left(1 \mathrm{~nm}=10^{-9} \mathrm{~m}\right)$, which appears violet, to $700 \mathrm{~nm}$, which appears red. Light outside this range of wavelengths exists, but we cannot see it [1].

Our eyes contain light-sensing cells called cones and rods. Both cones and rods respond to light, but they have different functions in vision. Rods are important in dim light, for example, at night, because they are highly sensitive to light. However, rods cannot detect colors. This is why the world looks black and white in moonlight. To see colors, we need cones. Cones are responsible for color vision, but they need bright light to work. Humans normally have three types of cones: blue (B), green $(\mathrm{G})$, and red (R). Each type of cone can detect a particular range of wavelengths: $\mathrm{B}$ cones respond most strongly to light that has a wavelength of about $420 \mathrm{~nm}, \mathrm{G}$ cones $534 \mathrm{~nm}$, and R cones $564 \mathrm{~nm}$ [1].

We can measure the strengths of the cones' responses to different wavelengths of light, and plot something called "spectral sensitivity curves," as you can see in the left panel of Figure 2A. For example, the spectral sensitivity of a human's $B$ cone peaks at $420 \mathrm{~nm}$, and becomes zero under about $380 \mathrm{~nm}$ and over about $550 \mathrm{~nm}$. In other words, a B cone can respond to light of $500 \mathrm{~nm}$, but only weakly. Bright $500 \mathrm{~nm}$ (green) light would produce the same response as dim $420 \mathrm{~nm}$ (blue) light in a B cone, which means that the B cone cannot distinguish bright $500 \mathrm{~nm}$ light from dim $420 \mathrm{~nm}$ light. If we only had $\mathrm{B}$ cones, we would be unable to see any color at all, only differences in brightness

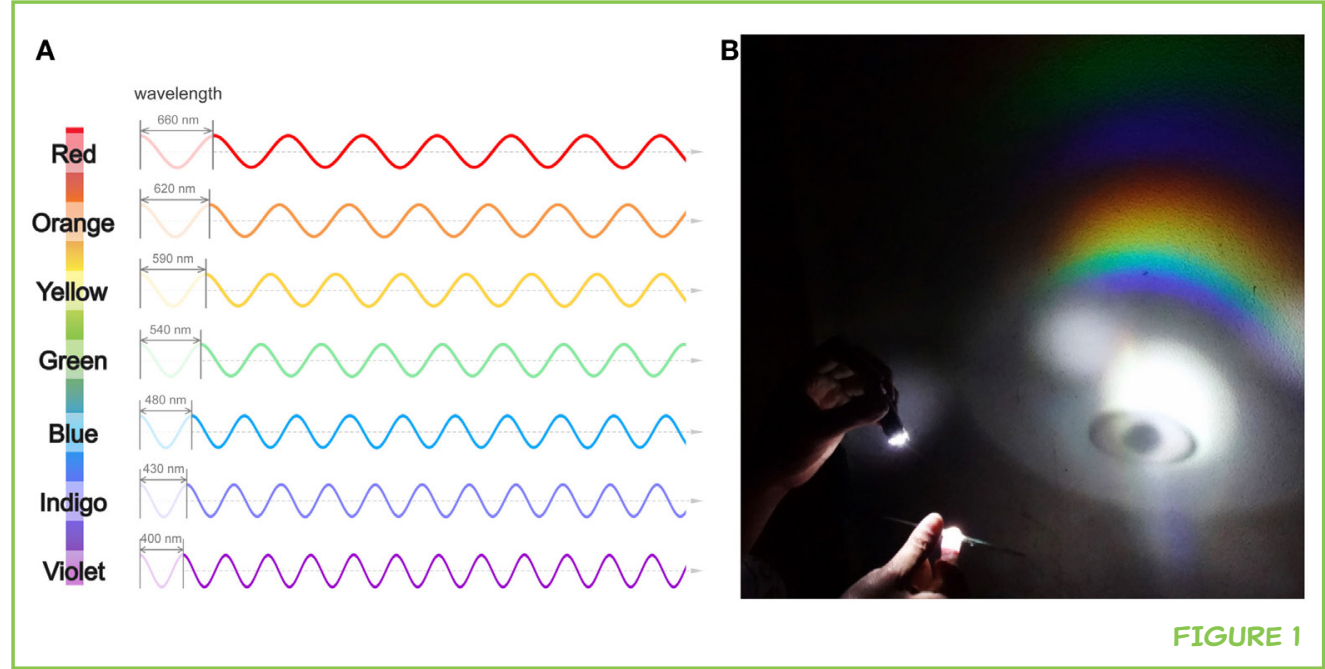




\section{FIGURE 2}

A. Light-sensing cells of humans (Homo sapiens), western honey bees (Apis mellifera), and blue tits (Cyanistes caeruleus). The graphs show spectral sensitivity curves of lightsensing cells, that is, how much the cells respond to light of different wavelengths. In humans (top), three light-sensing cells (called "cone" photoreceptors in vertebrates, because of the cells' shape) are used for seeing colors. Blue (B), green (G), and red $(R)$ cones have maximum sensitivity at 420, 534, and $564 \mathrm{~nm}$, respectively. Honey bees (middle) do not have lightsensing cells tuned to red, but instead have cells that are most sensitive to ultraviolet (UV) light. Blue tits (bottom) have four light-sensing cells. B. For humans with three types of light-sensing cells in the eye (trichromatic; "tri-" means three), it is easy to pick out the painted lady butterfly (Vanessa cardui) and small violet rosemary flowers (Rosmarinus officinalis) against the green vegetation. However, if an animal has only one type of lightsensing cell (monochromatic; "mono" means one), the butterfly and the flowers are harder to see. C. UV-reflective patterns on the large grass yellow butterfly, Eurema hecabe. Left pictures were taken with a regular camera, designed to match human color vision. Right pictures were taken with the same camera, but through a filter that only allows UV light to pass (photographs: Yuh-Tyng Lin).
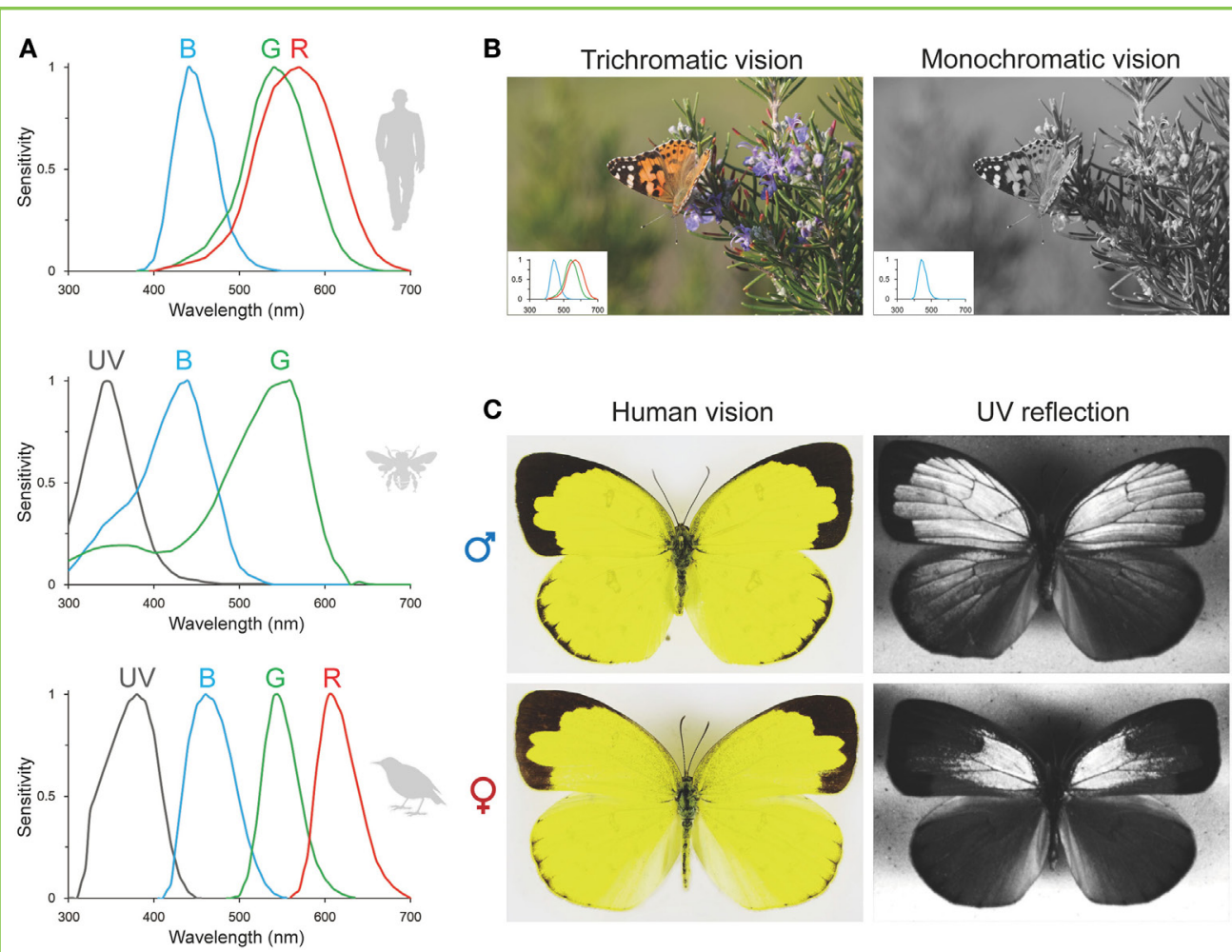

FIGURE 2

(Figure 2B). To see colors, an animal relies on a process known as comparison. The brain processes color information by comparing the outputs from two or more types of cones. To be able to distinguish colors, the eye has to have at least two different light sensors.

So, this is how we can tell whether other animals could have color vision. Of course, we cannot ask animals to describe the colors they see, as we can with humans, but we can design experiments that look at animal behavior, and these experiments can test the animal's color vision. This is a tricky process though, and color vision has been found successfully in only a few animal species, because these experiments are difficult. Often it is easier to check whether the eyes of the animal contain two or more different classes of light sensors, because we know that having at least two kinds of sensors potentially allows an animal to distinguish colors. To identify which light-sensing cells an animal has, we perform "electrophysiology": we measure the electrical signals that send information from the eye to the brain. We first insert an electrode into a light-sensing cell. Next, we shine a series of monochromatic lights (lights of very limited ranges of wavelengths) onto the eye, e.g., from 300 to $700 \mathrm{~nm}$ in $10 \mathrm{~nm}$ steps (i.e., $300,310,320$, up to $700 \mathrm{~nm}$ ). Then we record the electrical activity of the cell in response to each wavelength of light. Finally, we can collect the responses of this cell across the whole spectrum of wavelengths. If we repeat this process again and again to record different cells, in the end, we can discover how many and which types of light sensors the animal has [2]. 
ELECTROPHYSIOLOGY

Measuring the electrical activity inside living organisms. Electrocardiography and electroencephalography are electrophysiological techniques used by doctors to examine humans.

\section{COMPOUND EYE}

The kind of eye that most insects and crustaceans have. It consists of many units called ommatidia. Each ommatidium has a lens with a bundle of light-sensing cells underneath.

\section{ANIMALS AND HUMANS HAVE DIFFERENT TYPES OF LIGHT-SENSING CELLS}

Do all animals have the same set of light-sensing cells that we do? Honey bees also have three light-sensing cell types similar to our cones, but they are shifted toward shorter wavelengths: ultraviolet (UV), blue, and green instead of blue, green, and red (Figure 2A). In fact, bees are not particularly attracted to red flowers, because they do not see red well. Like some "color blind" people, bees have trouble distinguishing between red and green colors. However, bees can see UV light patterns that are invisible to us. Many flowers have UV patterns on their petals, which act as a signal to help bees and other insects to find and pollinate the flower. In addition, some birds and insects have UV patterns too, which the animals use to recognize each other. For example, as Figure $2 \mathrm{C}$ shows, the male and female yellow butterflies look almost identical to our eyes. However, if you take pictures with a camera only sensitive to UV light, you can clearly see different patterns on the wings. Because bees have UV-sensing cells but no red-sensing cells (Figure 2A), they probably see the world very differently than we do.

Not all animals have three types of light-sensing cells like humans and bees. For example, many birds have four: UV, B, G, and R cones (Figure 2A). These birds can see a broad range of wavelengths, extending from UV to red. They are also likely to be better than us at telling apart colors that are only slightly different. Some birds change the sensitivity of their cones to different wavelengths in the spectrum by using colored oil droplets inside their eye. These oil droplets work in a similar way to sunglasses with colored lenses. Some insects with compound eyes also do this [2].

Butterflies are another example of an animal with complex color vision. Their color vision system appears to have evolved from a three-color system based on UV-, B-, and G-sensing cells, like that of bees. Over millions of years, the color vision of some butterflies became more complex by adding extra lightsensing cells with different spectral sensitivities, probably to help them find flowers from which to drink nectar. For example, the Japanese yellow swallowtail (scientific name Papilio xuthus) has six types of light-sensing cells: UV, violet (V), B, G, R, and "broadband" (BB) (Figure 3A). The BB cells got that name because they respond to a wide variety of different wavelengths, rather than one specific color [3]. We recently studied the common bluebottle butterfly, Graphium sarpedon, and found that it has at least 15 different classes of light-sensing cells in the eye [4] (Figure 3B). This is the highest number of different kinds of light-sensing cells ever identified in an insect.

In some butterflies, the spectral sensitivities of the light-sensing cells even differ between the sexes of one species, which is called "sexual dimorphism." In the small white butterfly, Pieris rapae, only females have violet-sensitive cells, while males instead have double-peaked blue-sensing cells (Figure 3C). 


\section{FIGURE 3}

Spectral sensitivities of light-sensing cells differ greatly between species. This figure shows four examples. A. The Japanese yellow swallowtail butterfly, Papilio xuthus, has six cell types. In addition to the sensitivity curves peaking at ultraviolet, violet, blue, green, and red, you can see one that is very broad (yellow line). B. The common bluebottle butterfly, Graphium sarpedon, has 15 kinds of light sensors. Many of the spectral sensitivity curves are overlapping, so you need to carefully follow each line to identify them all. C. The small white butterfly, Pieris rapae, has seven cell types. Males and females of this species have different eyes; the dashed curves indicate cell types that are found in only one sex.

D. The mantis shrimp, Haptosquilla trispinosa has 16 kinds of cells, 11 of which are shown here. Compared to $G$. sarpedon, the curves are narrower and more evenly spaced, so maybe the shrimp brain processes color differently (D.; Thoen et al., 2014; photograph: Roy Caldwell).
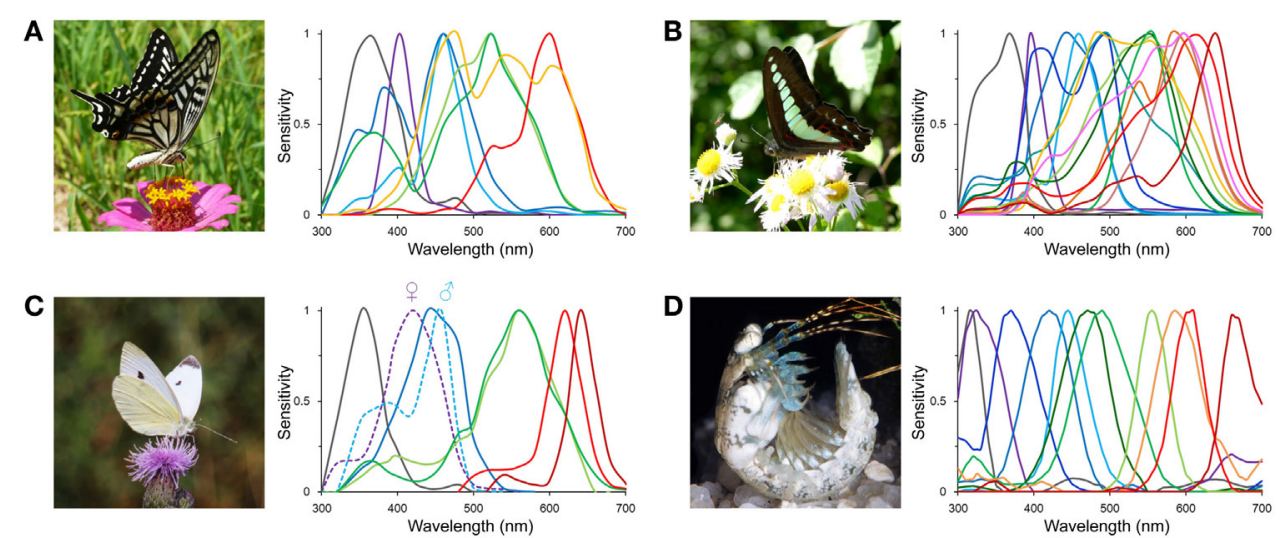

FIGURE 3

In the case of the sulfur butterfly, Colias erate, sexual dimorphism is found in the number of red-sensing cells: females have three types that are sensitive to slightly different shades of red, while males only have one type [5].

\section{THE MORE THE BETTER?}

You may be asking yourself: why do butterflies need so many types of lightsensing cells? Does having more types result in better color vision? Let's take a look at some examples. As we already discussed, most humans have three classes of cones. But some people (usually males) only have two fully functional cone types, with reduced sensitivity in the third type. We call this condition color blindness [6]. As we have seen, having just two cone types is enough for color vision. This means that most "color blind" people can still see lots of colors. It may be better to call these people color deficient instead of color blind. If someone color deficient and someone with normal vision view the same scene, they may see rather different things; color deficient people usually have difficulty in telling apart red from green. In other words, having fewer classes of light-sensing cells limits the ability to see colors. On the other hand, some people have four cone types. This is very rare and is only found in females. These people are thought to see a lot more colors than people with regular vision. The extra cone allows them to see additional colors that are invisible to most of us [7]. It seems that more light-sensing cells result in better color vision.

So far, the richest variety of light-sensing cells that has been found in the animal kingdom is in the eyes of reef-dwelling mantis shrimp, which have up to 16 types (Figure 3D) [5]. One might assume that these mantis shrimp have better color vision than any other animal. But in reality, their color vision turns out to be surprisingly poor [8]. Humans can distinguish between light wavelengths that differ by as little as $1 \mathrm{~nm}$. However, mantis shrimp can barely see wavelength differences of $15 \mathrm{~nm}$. It seems that mantis shrimp brains process color information in a different way than this information is processed in the brains 
of other animals. Some researchers think mantis shrimp might not compare the input from all their different light-sensing cell types the same way other animals do, but instead they take some processing "shortcut" to detect color quickly but not as accurately.

The Japanese yellow swallowtail butterfly, P. xuthus, has good color vision; it is able to detect wavelength differences of about $1 \mathrm{~nm}$, which is similar to human performance. Their eyes have at least six different types of lightsensing cells, but do they use all of them for color vision? This question has been answered by training the butterflies to detect the difference between two lights of slightly different wavelengths, and making a computer simulation to predict how the butterflies would perform if various combinations of light-sensing cells were used. The computer simulation indicated that their color vision is mainly based on the UV-, B-, G-, and R-sensing cells, suggesting that their color vision is a four-color system, like birds have. The other two classes of light-sensing cells, $\mathrm{V}$ and $\mathrm{BB}$, are probably not used for seeing colors, but instead used for some other visual functions. Perhaps butterfly vision functions similarly to human vision, with their UV-, B-, G-, and R-sensing cells functioning like cones and the BB- and/or V-sensing cells functioning like rods.

Is it a case of "the more, the better" when it comes to light-sensing cells? Do you think that the common bluebottle butterfly ( $G$. sarpedon) with 15 classes of light-sensing cells in the eye (Figure 3B) has better color vision than we do? We do not know yet. The answer can only be found through future experiments looking at butterfly behavior. Nevertheless, from the information about color vision that we have learned from humans and butterflies, it seems unlikely that the bluebottles use all 15 light-sensing cells just for seeing colors. We believe that they probably use only four of them for color vision as in Papilio, a close relative of the bluebottles. Compared to human vision, adding UV sensors certainly expands the range of visible light into the UV wavelength region, broadening the spectrum of colors than can be seen. The other 11 cell types may be useful to detect specific stimuli, for example, fast-moving objects against the sky, or particular light wavelengths reflected from potential mates or enemies.

\section{HOW CAN WE USE THE RESEARCH IN THE FUTURE?}

It is impossible for us to see the world as butterflies do; our eyes and brains lack the necessary equipment. But, it is still worthwhile and exciting to try to understand what a butterfly's visual experience is like by combining evidence from various kinds of experiments. We are also interested in how color vision systems have evolved over time by comparing the color vision of many different insects. In this process, we may even discover principles that we can use to build better "visual" systems for artificial intelligence. Birds and insects rely on their sense of sight to guide their flight, finding food and avoiding obstacles 
and predators. Imagine if we could program aerial drones to use their "eyes" (cameras) to pilot themselves as skillfully as animals do!

Another possible use of our research on insect vision is for farming. Nowadays, a lot of chemical insecticides are used to protect our crops. Unfortunately, these chemicals are often damaging to the environment, and insect pests can become resistant to them. Perhaps it is possible to keep insects away from crops cleanly and safely by using light instead of chemicals. In order to do this, we first have to understand how insects' eyes work.

\section{ORIGINAL SOURCE ARTICLE}

Chen, P.-J., Awata, H., Matsushita, A., Yang, E.-C., and Arikawa, K. 2016. Extreme spectral richness in the eye of the common bluebottle butterfly, Graphium sarpedon. Front. Ecol. Evol. 4:1-12. doi:10.3389/fevo.2016.00018.

\section{REFERENCES}

1. Land, M. F., and Nilsson, D.-E. 2002. Animal Eyes. Oxford: Oxford University Press.

2. Cronin, T. W., Johnsen, S., Marshall, N. J., and Warrant, E. J. 2014. Visual Ecology. Princeton, Oxford: Princeton University Press.

3. Arikawa, K. 2003. Spectral organization of the eye of a butterfly, Papilio. J. Comp. Physiol. A 189, 791-800. doi:10.1007/s00359-003-0454-7

4. Chen, P.-J., Awata, H., Matsushita, A., Yang, E.-C., and Arikawa, K. 2016. Extreme spectral richness in the eye of the common bluebottle butterfly, Graphium sarpedon. Front. Ecol. Evol. 4:1-12. doi:10.3389/fevo.2016.00018

5. Marshall, J., and Arikawa, K. 2014. Unconventional colour vision. Curr. Biol. 24, R1150-R1154. doi:10.1016/j.cub.2014.10.025

6. Backhaus, W. G. K., Kliegl, R., and Werner, J. S. 1998. Color Vision: Perspectives from Different Disciplines. Berlin, New York: Walter de Gruyter.

7. Jordan, G., Deeb, S. S., Bosten, J. M., and Mollon, J. D. 2010. The dimensionality of color vision in carriers of anomalous trichromacy. J Vis 10, 12. doi:10.1167/10.8.12

8. Thoen, H. H., How, M. J., Chiou, T. H., and Marshall, J. 2014. A different form of color vision in mantis shrimp. Science 343, 411-413. doi:10.1126/science.1245824

SUBMITTED: 12 July 2017; ACCEPTED: 06 December 2017;

PUBLISHED ONLINE: 09 January 2018.

EDITED BY: Wayne Iwan Lee Davies, University of Western Australia, Australia

CITATION: Chen P-J, Stewart FJ and Arikawa K (2018) The More, the Better? A Butterfly with 15 Kinds of Light Sensors in Its Eye. Front. Young Minds 5:70. doi:10.3389/ frym.2017.00070 
CONFLICT OF INTEREST STATEMENT: The authors declare that the research was conducted in the absence of any commercial or financial relationships that could be construed as a potential conflict of interest.

COPYRIGHT (C) 2018 Chen, Stewart, and Arikawa. This is an open-access article distributed under the terms of the Creative Commons Attribution License (CC BY). The use, distribution or reproduction in other forums is permitted, provided the original author(s) or licensor are credited and that the original publication in this journal is cited, in accordance with accepted academic practice. No use, distribution or reproduction is permitted which does not comply with these terms.

\section{REVIEWED BY}
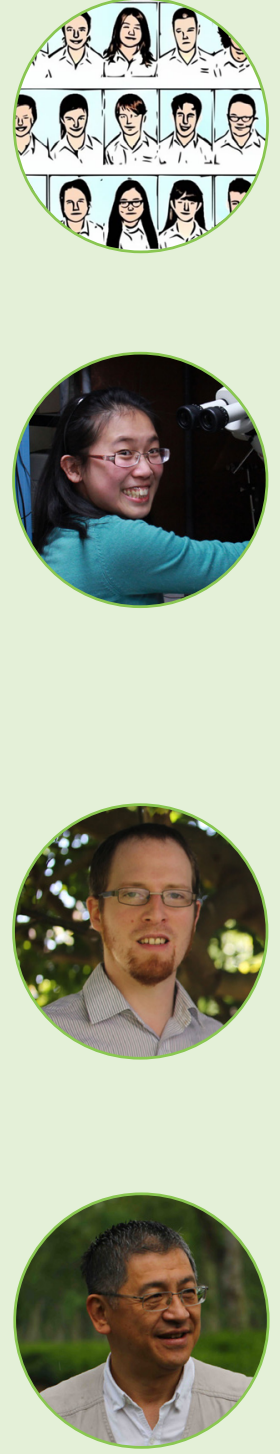

\section{MELVILLE SENIOR HIGH SCHOOL, AGE: 13-14}

A quirky group of science loving individuals who for the last 2 years have been perfecting their craft together. Embracing the term nerds, this class love to explore all things science.

\section{AUTHORS}

\section{PEI-JU CHEN}

A PhD student at Sokendai, graduated from National Taiwan University in 2012 with Master's degree in entomology (the study of insects). She originally comes from Taiwan, a small island that is extremely rich in butterflies. Butterflies feed on flowers and have colorful wings, so she is interested in the evolution of their color vision. She has studied several butterflies' visual systems from the perspective of brain science. Her current project aims to understand how the butterfly brain processes color information after light is detected by various light sensors in the eye.

\section{FINLAY J. STEWART}

Assistant professor of computational neuroethology (the study of how brains control behavior) at Sokendai, graduated from the University of Edinburgh, UK. In 2009, he completed his $\mathrm{PhD}$ on how fruit flies use their senses of sight and smell to find food. Since 2012, he has been studying the visual systems of butterflies. Originally from a computer science background, he is interested in using computational techniques to investigate how insect brains work, mostly through studying insect behavior.

\section{KENTARO ARIKAWA}

Professor of neuroethology at Sokendai, graduated from Jiyu-Gakuen College and Sophia University graduate school. As a first-year graduate student, he discovered that butterflies can detect light with their genitals and investigated the function of this unique light-sensing system during his PhD research. After being a biology professor at Yokohama City University for 21 years, he moved to Sokendai in 2006. He also served as a research fellow at Australian National University, Mitsubishi-Kasei Institute of Life Science, and the National Institutes of Health, and as a researcher of JST-PRESTO. *arikawa@soken.ac.jp 